\title{
Associação entre coleta de resíduos sólidos domiciliares e saúde, Belo Horizonte (MG), Brasil
}

\author{
Cícero Antônio Antunes Catapreta ${ }^{1}$ e Léo Heller ${ }^{1}$
}

\begin{abstract}
RESUMO Existem poucos estudos publicados sobre o efeito da coleta inadequada de resíduos sólidos sobre a saúde da população exposta a estes resíduos. O objetivo do presente trabalho foi descrever esta associação em uma amostra de crianças menores de 5 anos, moradoras de sete vilas e favelas em Belo Horizonte, Minas Gerais, Brasil. Foram consideradas expostas as crianças cujas famílias não eram beneficiadas pela coleta; não expostas eram as crianças cujas famílias moravam em zonas de coleta. Foi empregado o delineamento epidemiológico seccional, sendo definidos como "casos" os registros ambulatoriais secundários que indicavam doenças diarréicas, parasitárias e dermatológicas. Paralelamente, as demais notificações observadas para a mesma faixa etária constituíram o grupo controle. O estudo foi realizado com base em dados de 1994, sendo que os dados sobre incidência das doenças que caracterizaram os casos, assim como os registros empregados para a composição da amostra de controles, foram obtidos junto ao sistema informatizado da Secretaria Municipal da Saúde. O estudo epidemiológico desenvolvido revelou associação entre ausência de coleta de resíduos sólidos domiciliares e saúde pública. Os resultados sugerem que a população infantil exposta à ausência de serviços de coleta dos resíduos sólidos domiciliares possui $40 \%$ (razão de possibilidades de ocorrência ou odds ratio da ordem de 1,40) mais oportunidade de apresentar doenças diarréicas, parasitárias e dermatológicas do que a população não exposta. Além disso, o cálculo do risco atribuível populacional revelou que a universalização da coleta de lixo poderia evitar, considerando o quadro existente em 1995, 512 casos entre crianças nas vilas e favelas estudadas e, para a situação existente em 1994, 2316 casos entre a população infantil em toda a cidade de Belo Horizonte.
\end{abstract}

A influência do manejo inadequado dos resíduos sólidos urbanos sobre a saúde humana tem despertado a aten-

\footnotetext{
1 Universidade Federal de Minas Gerais, Departamento de Engenharia Sanitária e Ambiental. Correspondência e pedidos de separatas devem ser enviados a Léo Heller no seguinte endereço: Departamento de Engenharia Sanitária e Ambiental, Universidade Federal de Minas Gerais, Avenida do Contorno 842, $7^{\circ}$ andar, Centro, CEP 30110-060, Belo Horizonte, MG, Brasil. Telefone: +55-31-2381880; fax: +55-31-238-1879; email: heller@adm.eng. ufmg.br
}

ção de diversas entidades e profissionais ligados ao saneamento, face à presença nas cidades de inúmeros locais de acúmulo de lixo que propiciam um triste quadro de degradação social e ambiental. Tais locais geralmente situam-se nas zonas periféricas dos centros urbanos, em áreas de baixo valor econômico, onde reside a população mais carente e mais vulnerável às conseqüências desse tipo de disposição dos resíduos sólidos. Trata-se geralmente de uma população desnutrida e, portanto, suscetível de contrair e transmitir doenças adquiridas pela prática da catação, para o que são atraídas (1), e pela exposição ambiental a que estão submetidas.

A ausência de coleta, associada ao manuseio e à disposição inadequados dos resíduos sólidos municipais, é importante fator de risco para a saúde pública $(2,3)$. No entanto, é reconhecido que são raras as informações concernentes aos efeitos dos resíduos sólidos sobre a saúde humana (4). A 
bibliografia disponível revela também pouca atenção à relação entre as formas de disposição final e a saúde.

O ser humano pode ser atingido de diversas formas pelos efeitos indesejáveis do lixo, em especial porque o lixo constitui ambiente favorável à atração e ao desenvolvimento de diversos animais e microrganismos veiculadores de doenças (5-7). No caso particular dos depósitos de lixo onde a disposição inadequada se faz presente, e onde é permitido o acesso de pessoas, os resíduos oferecem potencial risco de contaminação, sobretudo quando as pessoas, juntamente com os animais que habitam esses locais, disputam os componentes da massa de resíduos, inclusive "alimentos".

Considerando a significativa lacuna na literatura sobre o tema, o objetivo do presente trabalho foi investigar o efeito da ausência de coleta de resíduos sólidos domiciliares sobre a saúde das crianças com idade inferior a 5 anos, residentes em sete vilas e favelas no município de Belo Horizonte, Estado de Minas Gerais, Brasil. Para tanto, foi analisada a presença de diarréia, parasitoses e doenças de pele em crianças residentes em zonas com e sem coleta pública de lixo, empregando delineamento epidemiológico especificamente concebido.

\section{MATERIAIS E MÉTODOS}

\section{Áreas investigadas}

A pesquisa avaliou dados de 1994 do município de Belo Horizonte, capital do estado de Minas Gerais, Brasil, cidade com população de cerca de 2100000 habitantes (em 1996). Foram estudadas sete vilas e favelas; são consideradas vilas os assentamentos precários, com razoável nível de urbanização, sendo os moradores, em geral, proprietários dos terrenos. As favelas são assentamentos com urbanização muito precária, em geral zonas onde os terrenos foram invadidos. As vilas e favelas estudadas se localizam na região periférica da cidade (figura 1), onde os serviços de coleta de resíduos
FIGURA 1. Vilas e favelas incluídas no estudo sobre associação entre ausência de coleta de lixo e saúde, Belo Horizonte, Minas Gerais, Brasil, 1994

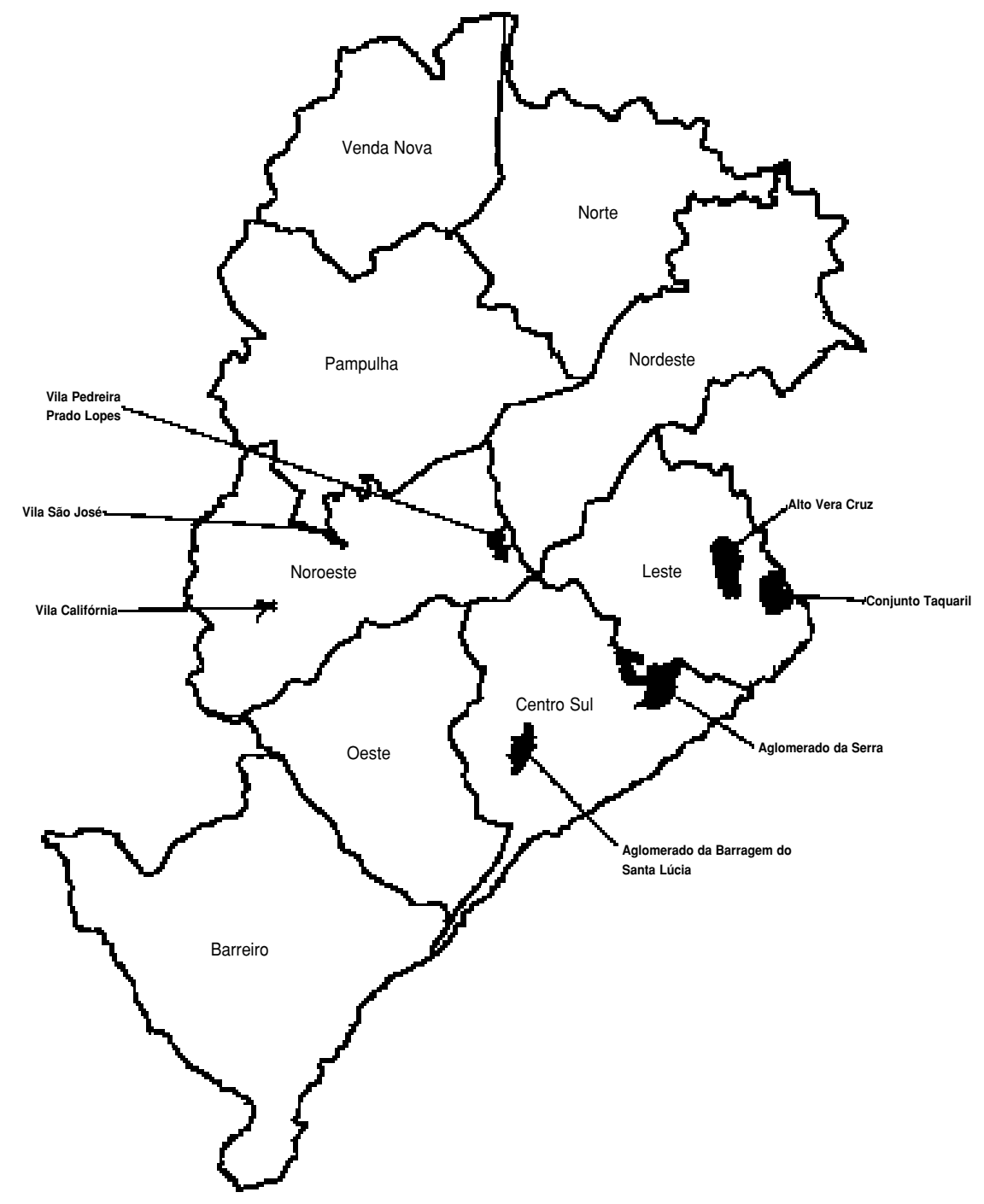

sólidos não atendem toda a população: Aglomerado da Serra (45920 habitantes); Aglomerado do Santa Lúcia (16835 habitantes); Alto Vera Cruz (30 075 habitantes); Conjunto Taquaril (20 500 habitantes); Vila Califórnia (3 610 habitantes); Vila Pedreira Prado Lopes (6 680 habitantes); e Vila São José (8115 habitantes). A população total para as sete regiões era de 131735 habitantes na época do estudo. Para a escolha das áreas incluídas na pesquisa, foram observados os seguintes critérios principais:
- número significativo de sujeitos potencialmente suscetíveis de contraírem as doenças pesquisadas;

- deficiência na cobertura por serviços de coleta de resíduos sólidos domiciliares;

- facilidade de acesso aos dados de morbidade pesquisados.

\section{Delineamento epidemiológico}

Foi adotado o delineamento epidemiológico do tipo seccional, no qual 
tanto os dados sobre exposição a resíduos sólidos quanto os dados sobre indicadores de saúde referem-se ao mesmo período, que no presente estudo equivale ao ano de 1994.

Diferentemente da estrutura clássica para esse tipo de delineamento, trabalhou-se com o conceito de "controles", a exemplo dos estudos de casocontrole. O grupo controle não foi definido da forma tradicional (total da amostra subtraída do grupo de casos), e sim como o grupo de pacientes notificados no sistema de saúde, com registros diferentes daqueles definidos para o grupo de casos.

Foram caracterizados como "casos" as crianças afetadas por doenças cujo ciclo de transmissão pode ter relação com o lixo inadequadamente disposto. O grupo "controle" incluiu as afecções à saúde sem nexo causal com a ausência de coleta.

Inicialmente, foram consideradas casos as crianças diagnosticadas com diarréia e parasitoses, conforme recomendação da literatura $(6,8-10)$. Porém, no intuito de diversificar os cenários, de forma que fosse possível estudar diferentes hipóteses de im- pacto sobre a saúde, num segundo momento as doenças dermatológicas também foram incluídas no grupo de indicadores $(2,11)$. Todas as crianças com menos de 5 anos com outros diagnósticos, no mesmo período, foram incluídas no grupo controle. Além disso, para fins de análise, um subgrupo controle foi definido, denominado de grupo de "selecionados". Neste subgrupo foram excluídos os registros que não se mostravam pertinentes ao estudo, conforme relação a seguir:

- sintomas, sinais e afecções mal definidos;

- outros efeitos de causas externas e os não especificados;

- consultas para supervisão de saúde da criança;

- consultas de planejamento familiar (procedimentos anticoncepcionais e relativos a procriação);

- indivíduos que apresentam condições que influenciam seu estado de saúde;

- indivíduos que entram em contato com serviços de saúde em outras circunstâncias, a fim de receberem procedimentos específicos, ou para acompanhamento;

- consultas para observação e avaliação de condições suspeitas;

- contatos para fins administrativos e atestados médicos em geral;

- exame ginecológico;

- exame preventivo de colo uterino;

- visitas por razões ignoradas.

Portanto, as seguintes definições foram consideradas para o grupo de casos: 1) casos diagnosticados com diarréia e parasitoses; 2) casos diagnosticados com diarréia, parasitoses e doenças dermatológicas. Para o grupo controle, quatro definições foram consideradas: 1) todos os registros notificados, exceto diarréia e parasitoses; 2) todos os registros notificados, exceto diarréia, parasitoses e doenças dermatológicas; 3 ) registros selecionados, exceto diarréia e parasitoses; 4 ) registros selecionados, exceto diarréia, parasitoses e doenças dermatológicas.

Para fins de análise, os grupos foram comparados da seguinte forma (figura 2): o grupo de casos conforme a definição 1 foi comparado aos controles definição 1 (combinação A); aos

FIGURA 2. Fluxograma das combinações entre as definições de caso e de controle, estudo sobre associação entre ausência de coleta de lixo e saúde, Belo Horizonte, Minas Gerais, Brasil, 1994

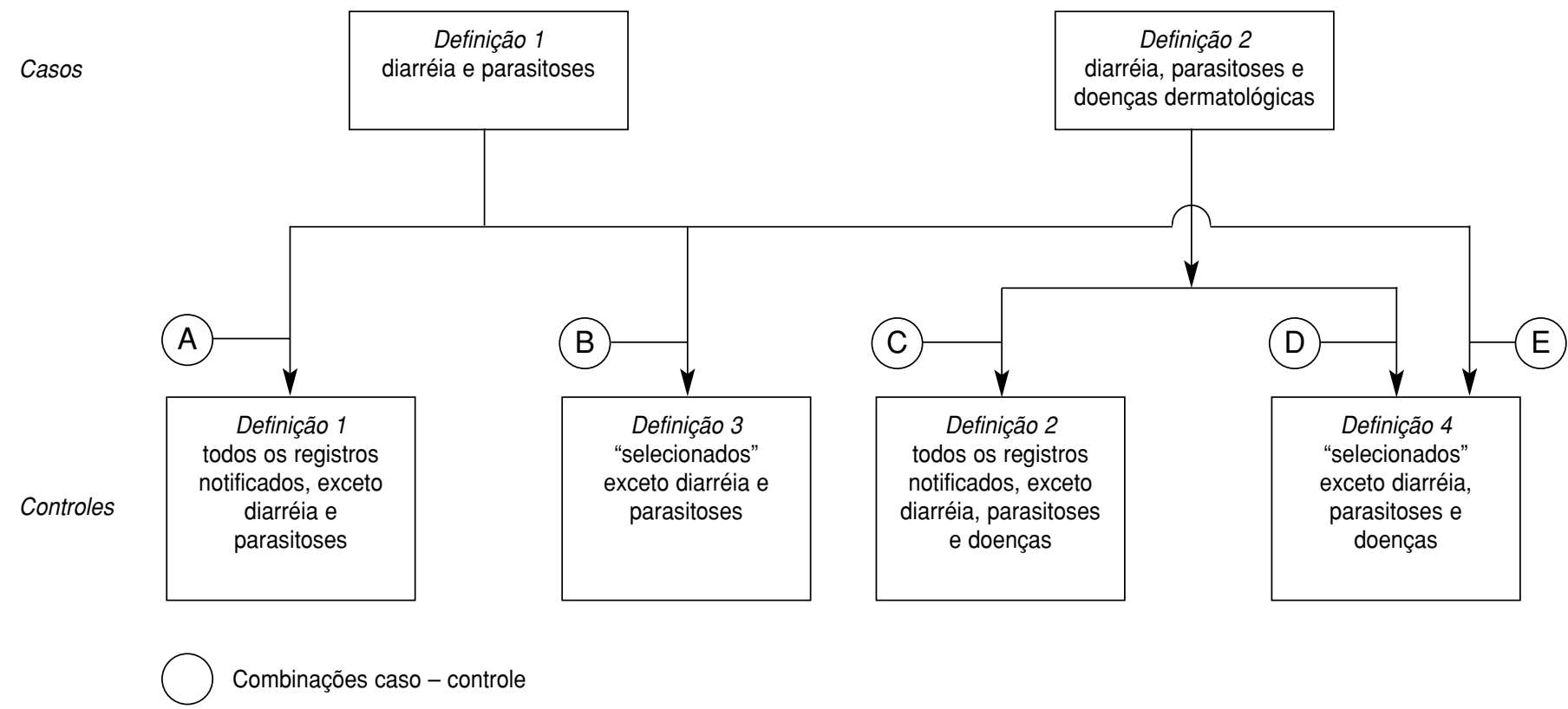


controles definição 3 (combinação B); e aos controles definição 4 (combinação E). O grupo de casos conforme a definição 2 foi comparado aos controles definição 2 (combinação C) e aos controles definição 4 (combinação D). Na combinação E optou-se por excluir as doenças dermatológicas da amostra.

\section{Amostragem}

Os dados populacionais para este estudo seccional foram coletados junto ao sistema informatizado da Secretaria Municipal de Saúde, que possui uma base de dados significativa e suficiente para a pesquisa desenvolvida. Este sistema de informações foi implantado em 1994, tendo como objetivo coletar e estudar dados amostrais de morbidade ambulatorial registrados nas unidades de saúde gerenciadas pela Secretaria Municipal de Saúde.

A Secretaria Municipal de Saúde realiza um inquérito quatro vezes ao ano, durante 1 semana a cada trimestre. As informações são coletadas pelos próprios funcionários das unidades de saúde. Além dos dados referentes à morbidade diagnosticada, são registradas informações sobre centro de saúde que prestou o atendimento; data de atendimento; código da Classificação Internacional de Doenças no qual se enquadra o diagnóstico; endereço, idade e sexo do paciente.

Após a coleta, as informações são digitadas nos distritos sanitários correspondentes. Durante este período, são verificadas possíveis inconsistências; se for constatada alguma falha, o registro é devolvido à unidade de saúde para reavaliação. Em seguida, procede-se à geocodificação do banco de dados, onde este é harmonizado com o sistema de geoprocessamento de dados do município, ou seja: as informações são preparadas para que possam ser fornecidas conforme diferentes interesses (por exemplo, por número de atendimentos em centro de saúde, por tipo de notificação, etc.).

Um total de 102467 registros foram coletados no inquérito anual realizado pela Secretaria Municipal de Saúde em 1994. No presente estudo, apenas crianças com até 5 anos foram selecionadas, totalizando 23998 registros em Belo Horizonte em 1994. Nas áreas de estudo, a amostra de crianças com menos de 5 anos foi de 2394; dentre estas, 554 foram perdidas. Portanto, um total de 1840 crianças (incluindo casos e controles) participaram do estudo.

\section{Definição das condições de exposição}

Definiu-se como "exposição" a ausência de coleta pública de resíduos sólidos domiciliares, supondo-se que a população submetida a esta situação encontra-se exposta tanto à ação de vetores que sobrevivem nos resíduos não coletados, quanto ao contato direto com os resíduos. Os locais onde havia coleta foram identificados por meio de consultas aos cadastros de roteiros de coleta da Superintendência de Limpeza Urbana de Belo Horizonte (SLU).

Como a coleta é feita de diversas formas (com caminhões compactadores, caminhões de carroceria aberta, caçambas estacionárias e veículos menores, tipo caminhonete), um critério foi adotado para delimitar as áreas de abrangência do serviço de coleta, obedecendo à definição a seguir: a área de abrangência dos serviços de coleta de lixo corresponde a uma faixa de 80 metros, a partir do eixo da rota da coleta regular, ou a um raio de 80 metros, a partir da localização das caçambas estacionárias.

Ficou estabelecido que todo indivíduo residente dentro da área de abrangência estabelecida segundo a definição acima era não exposto à ausência de coleta. No entanto, como nessas regiões a coleta de resíduos sólidos domiciliares é precária, acarretando presença de pontos de confinamento, pequenos depósitos de lixo, critério mais específico foi adotado. Sendo assim, nestes casos, considerou-se um círculo de influência desses pontos, com raio de $80 \mathrm{~m}$, assumido como região não atendida. Adotou-se como limite da área de influência a distância média entre os dois extremos da região de intersecção entre o círculo de influência dos depósitos e a área de abrangência da coleta.

\section{Potenciais fatores de confusão}

Como o objetivo da pesquisa foi avaliar a associação entre ausência de coleta de resíduos sólidos domiciliares e a incidência de certas doenças, outros fatores que podem intervir nesta associação devem ser analisados. Fatores ambientais, socioeconômicos e ligados ao comportamento são usualmente apontados como possíveis variáveis de confusão em tal tipo de estudo. Nesta investigação, considerou-se que a própria seleção das áreas já é um procedimento que torna homogêneos os grupos segundo algumas variáveis ambientais, como abastecimento de água e perfil socioeconômico. Certos fatores ligados ao comportamento podem estar relacionados à condição socioeconômica e assim teriam sido controlados por meio da seleção das áreas. Porém, aqueles não relacionados a tal condição dificilmente seriam controlados, já que o estudo se baseia em dados secundários.

Um importante fator ambiental de confusão para o tipo de associação pesquisada, o qual foi investigado neste estudo, é o atendimento por esgoto sanitário. A ausência ou precariedade deste serviço pode vir a influenciar os resultados, uma vez que os indicadores de saúde analisados também podem estar a ele associados. Além disso, é uma variável associada à coleta de resíduos sólidos, em vista das características urbanísticas das vilas e favelas, que favorecem ou não tanto a implantação de serviços de coleta de lixo quanto a implantação de redes de esgoto sanitário.

A Companhia de Saneamento de Minas Gerais (COPASA-MG) forneceu informações referentes ao sistema de esgoto sanitário nas áreas pesquisadas. Porém, a COPASA-MG não possui cadastro de redes coletoras de esgoto clandestinas, ou seja, implantadas sem a intervenção do poder público, o que é comum nas regiões estudadas.

Em vista disso, foi consultada a Secretaria de Estado do Trabalho e Ação 
Social (SETAS), que de 1983 a 1992 desenvolveu um programa de melhorias urbanas em vilas e favelas da região metropolitana de Belo Horizonte. Através desta consulta foi possível montar um cadastro abrangente sobre o sistema de esgoto sanitário clandestino de algumas áreas envolvidas.

\section{Medidas de risco}

Três medidas de risco foram empregadas na análise dos dados:

- Razão de possibilidades de ocorrência, ou odds ratio $(O R)$, determinada a partir da tabela $2 \times 2$, comumente empregada em estudos epidemiológicos. Valores de $O R$ e respectivo intervalo de confiança (IC) superiores a 1 significam que a associação entre exposição e doença é estatisticamente significativa.

- Risco atribuível populacional (RAP), que estima a queda percentual no número de casos da doença, caso a exposição seja eliminada. Esta medida foi calculada através da fórmula

$$
\mathrm{RAP} \%=\frac{P(O R-1)}{[P(O R-1)+1]},
$$

onde $P$ é a proporção de expostos na amostra populacional estudada $(12,13)$.

- Método de Mantel-Haenszel, ou odds ratio de Mantel-Haenszel $\left(O R_{\mathrm{MH}}\right)$, utilizado para avaliar a possível influência de variáveis confundíveis (14). Considerou-se que a relação $O R / O R_{\mathrm{MH}}$ compreendida na faixa de 0,90 a 1,10 indica que a variável não é fator de confusão.

\section{RESULTADOS}

Do universo populacional estudado (total de 2394 crianças e, excluindo-se as perdas, 1840 crianças) a amostra de casos abrangeu 246 crianças, observando a definição 1, e 378 crianças para a definição 2. A tabela 1 apresenta a distribuição da amostra de casos, por região estudada. A tabela 2 apresenta a distribuição por região do grupo
TABELA 1. Número de casos de diarréia, parasitoses e doenças da pele, segundo região estudada, Belo Horizonte (MG), Brasil, 1995

\begin{tabular}{lccccc}
\hline \multicolumn{1}{c}{ Vila/Favela } & Diarréia & Parasitoses & $\begin{array}{c}\text { Doenças } \\
\text { da pele }\end{array}$ & $\begin{array}{c}\text { Diarréias e } \\
\text { parasitoses }\end{array}$ & $\begin{array}{c}\text { Diarréias, } \\
\text { parasitoses e } \\
\text { doenças da pele }\end{array}$ \\
\hline Aglomerado da Serra & 32 & 75 & 65 & 107 & 172 \\
Aglomerado do Santa Lúcia & 14 & 27 & 16 & 41 & 57 \\
Alto Vera Cruz & 18 & 23 & 25 & 41 & 66 \\
Conjunto Taquaril & 13 & 26 & 14 & 39 & 53 \\
Vila Califórnia & 1 & - & 3 & 1 & 4 \\
Vila Pedreira Prado Lopes & 3 & 5 & 6 & 8 & 14 \\
Vila São José & 4 & 5 & 3 & 9 & 12 \\
Total & 85 & 161 & 132 & 246 & 378 \\
\hline
\end{tabular}

TABELA 2. Distribuição do grupo controle segundo região e definição, estudo sobre associação entre coleta de lixo e saúde, Belo Horizonte (MG), Brasil, 1995

\begin{tabular}{lrrrr}
\hline & \multicolumn{4}{c}{ Definição do grupo controle } \\
\cline { 2 - 5 } \multicolumn{1}{c}{ Vila/Favela } & \multicolumn{1}{c}{ ( } & \multicolumn{1}{c}{3} & 4 \\
\hline Aglomerado da Serra & 531 & 466 & 380 & 315 \\
Aglomerado do Santa Lúcia & 290 & 274 & 202 & 186 \\
Alto Vera Cruz & 365 & 339 & 256 & 230 \\
Conjunto Taquaril & 252 & 239 & 184 & 171 \\
Vila Califórnia & 24 & 21 & 15 & 12 \\
Vila Pedreira Prado Lopes & 73 & 67 & 34 & 28 \\
Vila São José & 59 & 56 & 46 & 43 \\
Total & 1594 & 1462 & 1117 & 985
\end{tabular}

a 1) Todos os registros, exceto diarréia e parasitoses; 2) todos os registros, exceto diarréia, parasitoses e doenças dermatológicas; 3) registros selecionados, exceto diarréia e parasitoses; 4) registros selecionados, exceto diarréia, parasitoses e doenças dermatológicas.

TABELA 3. Perdas observadas na seleção de casos e controles, conforme motivo e região, estudo sobre associação entre coleta de lixo e saúde, Belo Horizonte (MG), Brasil, 1995

\begin{tabular}{|c|c|c|c|c|c|c|c|}
\hline \multirow[b]{2}{*}{ Vila/Favela } & \multicolumn{7}{|c|}{ Motivo da perda } \\
\hline & $\begin{array}{l}\text { Doença } \\
\text { ignorada }\end{array}$ & $\begin{array}{l}\text { Idade } \\
\text { ignorada }\end{array}$ & $\begin{array}{c}\text { Idade e } \\
\text { doença } \\
\text { ignoradas }\end{array}$ & $\begin{array}{c}\text { Problemas } \\
\text { no } \\
\text { geoproces- } \\
\text { samento }\end{array}$ & $\begin{array}{c}\text { Total } \\
\text { perdas }\end{array}$ & $\begin{array}{l}\text { Amostra } \\
\text { total }\end{array}$ & $\begin{array}{c}\% \\
\text { perdas }^{a}\end{array}$ \\
\hline $\begin{array}{l}\text { Aglomerado da Serra } \\
\text { Aglomerado do }\end{array}$ & 4 & 30 & 1 & 74 & 109 & 747 & 14,59 \\
\hline Santa Lúcia & 1 & 21 & - & 27 & 49 & 380 & 12,89 \\
\hline Alto Vera Cruz & 7 & 25 & 3 & 119 & 154 & 560 & 27,50 \\
\hline Conjunto Taquaril & 14 & 26 & - & 146 & 186 & 477 & 38,99 \\
\hline $\begin{array}{l}\text { Vila Califórnia } \\
\text { Vila Pedreira Prado }\end{array}$ & - & 7 & - & - & 7 & 32 & 21,88 \\
\hline Lopes & 3 & - & - & 38 & 41 & 122 & 33,61 \\
\hline Vila São José & 1 & 6 & - & 1 & 8 & 76 & 10,53 \\
\hline Total & 30 & 115 & 4 & 405 & 554 & 2394 & 23,14 \\
\hline
\end{tabular}

${ }^{a}$ Em relação à amostra total. 
TABELA 4. Razão de possibilidades de ocorrência $(O R)$ bruta de diarréia, parasitoses e doenças dermatológicas para cada região em função das combinações entre casos e controles, estudo sobre associação entre coleta de lixo e saúde, Belo Horizonte (MG), Brasil, 1995

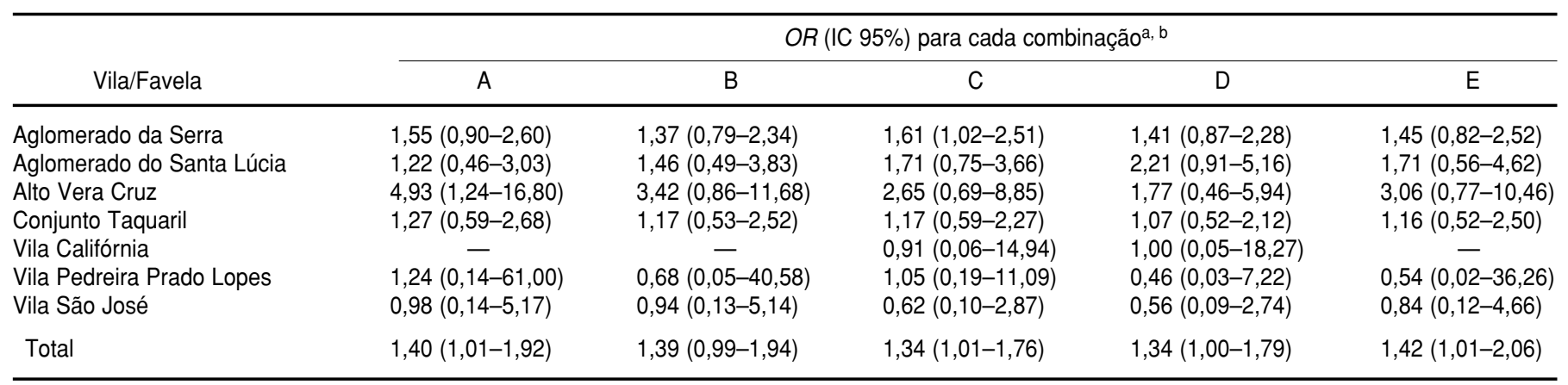

${ }^{a}$ A) Registros de diarréia e parasitoses versus todos os registros notificados, exceto diarréia e parasitoses; B) registros de diarréia e parasitoses versus registros selecionados, exceto diarréia e parasitoses; C) registros de diarréia, parasitoses e doenças dermatológicas versus todos os registros, exceto diarréia, parasitoses e doenças dermatológicas; D) registros de diarréia, parasitoses e doenças dermatológicas versus registros selecionados, exceto diarréia, parasitoses e doenças dermatológicas; E) registros de diarréia e parasitoses versus registros selecionados, exceto diarréia, parasitoses e doenças dermatológicas.

${ }^{b}$ Valores de $O R$ superiores a 1 significam que a associação entre exposição e doença é estatisticamente significativa.

controle, conforme as quatro definições consideradas.

Na seleção da amostra foram verificadas perdas nos grupos de casos e controles. As perdas foram ocasionadas, em sua maioria, porque não foi possível localizar os endereços pelo sistema de geocodificação, devido a problemas de interpretação dos dados pelo programa. Alguns endereços apresentaram-se inconsistentes, outros não puderam ser identificados pelo programa porque a base cartográfica utilizada é de 1989, portanto desatualizada. Além disso, alguns registros apresentavam idade ou diagnóstico ignorados. A tabela 3 apresenta o número e o percentual de perdas, segundo o motivo.

Na tabela 4 são apresentados os resultados do $O R$, com seu respectivo intervalo de confiança em um nível de significância de 5\%, para as combinações analisadas. Para a determinação dos $O R$ e respectivos intervalos de confiança, consideraram-se tabelas de contingência $2 \times 2$, comparando o número de casos expostos e de controles não expostos com o número de casos não expostos e de controles expostos. A tabela 5 mostra esta comparação para a amostra total na combinação A. Como pode ser observado, os valores totais encontrados são significativos, mostrando que, em geral, a população não atendida por coleta de resíduos sólidos domiciliares está exposta a um risco aproximadamente 1,4 vezes maior para as doenças investigadas do que a população provida dos serviços de coleta.

A possível influência da variável atendimento por serviços de esgoto sanitário como fator de confusão foi avaliada por análise estratificada. Esta
TABELA 5. Determinação da razão de possibilidades de ocorrência $(O R)$ bruta para a amostra total na combinação $A$, estudo sobre associação entre coleta de lixo e saúde, Belo Horizonte (MG), Brasil, 1995

\begin{tabular}{lccr}
\hline & Casos & Controles & Total \\
\hline Expostos & 64 & 320 & 384 \\
Não expostos & 182 & 1274 & 1456 \\
Total & 246 & 1594 & 1840 \\
\hline
\end{tabular}

a Registros de diarréia e parasitoses (casos) e todos os registros notificados, exceto diarréia e parasitoses (controles); $O R=1,40$. análise foi desenvolvida para a amostra total e para as regiões do Aglomerado da Serra e Aglomerado do Santa Lúcia, já que a relação exposto/não exposto a sistema de esgoto nestas áreas possibilitou a referida análise. A tabela 6 apresenta os resultados obtidos.

Como pode ser observado, os valores referentes à amostra total mostraram-se compreendidos na faixa descrita $\left(O R / O R_{\mathrm{MH}}=0,90-1,10\right)$, sugerindo que esgoto não deve ser considerado fator de confusão. Além disso, os valores do $O R_{\mathrm{MH}}$ e seu IC são muito próximos do $O R$ bruto, indicando que esgoto sanitário não influi sobre risco à saúde decorrente da ausência da coleta de resíduos sólidos domiciliares.

Os valores do risco atribuível populacional foram estimados para os casos representados pelas diarréias, parasitoses e doenças dermatológicas e controles selecionados, segundo a combinação E. $\mathrm{O}$ valor de $P$ adotado para Belo Horizonte foi de 0,15 (15\%), em função do atendimento por coleta de resíduos sólidos domiciliares pela SLU no ano de 1994. Para as vilas e favelas estudadas, o valor de $P$ foi calculado em 0,3626 . Este valor corresponde à parcela da população residente nestas áreas $(36,26 \%)$ e não atendida por serviços de coleta de resíduos sólidos, conforme dados de 1995. A extrapolação dos dados revela que vários casos inerentes aos indicadores adotados poderiam ser evitados (tabela 7). 
TABELA 6. Resultados da análise estratificada para sistema de esgoto como fator de confusão em função das combinações ${ }^{a}$ entre casos e controles, estudo sobre associação entre coleta de lixo e saúde, Belo Horizonte (MG), Brasil, 1995

\begin{tabular}{lccccc}
\hline & \multicolumn{5}{c}{$O R_{\text {BRUTO }} / O R_{\mathrm{MH}}$} \\
\cline { 2 - 6 } \multicolumn{1}{c}{ Vila/Favela } & $\mathrm{A}$ & $\mathrm{B}$ & $\mathrm{C}$ & $\mathrm{D}$ & $\mathrm{E}$ \\
\hline Aglomerado da Serra & 1,17 & 1,17 & 1,17 & 1,14 & 1,14 \\
Aglomerado Santa Lúcia & 1,06 & 1,00 & 1,06 & 1,27 & 1,22 \\
Amostra Total & 1,04 & 1,00 & 0,99 & 1,02 & 0,96 \\
\hline
\end{tabular}

${ }^{a}$ A) Registros de diarréia e parasitoses versus todos os registros notificados, exceto diarréia e parasitoses; B) registros de diarréia e parasitoses versus registros selecionados, exceto diarréia e parasitoses; C) registros de diarréia, parasitoses e doenças dermatológicas versus todos os registros, exceto diarréia, parasitoses e doenças dermatológicas; D) registros de diarréia, parasitoses e doenças dermatológicas versus registros selecionados, exceto diarréia, parasitoses e doenças dermatológicas; E) registros de diarréia e parasitoses versus registros selecionados, exceto diarréia, parasitoses e doenças dermatológicas.

\section{DISCUSSĀO}

A análise univariada indicou a presença de risco para a população exposta, conforme pode ser observado na tabela 4 . Os resultados foram significativos, tendo os valores de OR se situado em torno de 1,40. Entre as cinco combinações analisadas (A, B, C, D e $E)$, apenas um resultado não mostrou significância estatística, embora estivesse bastante próximo (limite inferior do IC $=0,99$ ). A consistência de resultados para o conjunto de combinações entre as definições de caso e controle sugere fortemente a presença de risco para a saúde na ausência de coleta de resíduos sólidos domiciliares nas vilas e favelas analisadas.

Estudo realizado em localidade próxima a Belo Horizonte sugeriu que o risco de diarréia para crianças cuja família se desfaz de seus resíduos de forma inadequada é aproximadamente $61 \%$ maior (risco relativo $=1,61$; IC95\%: 1,11-2,34) do que para as crianças cujas famílias são beneficiadas com coleta de resíduos (15). Por outro lado, em estudo visando verificar a associação entre disposição inadequada de resíduos sólidos e incidência de diarréia em crianças com idade inferior a 5 anos, foi encontrado um $O R$ de 3,98; IC95\%: 1,56-10,13, sugerindo a presença de elevado risco (16). Em outro estudo (17), ficou demonstrado que crianças com menos de 5 anos de idade, residentes em áreas beneficiadas com coleta regular ou irregular de resíduos sólidos, apresentavam 65\% menos episódios de diarréia do que aquelas que residiam em locais sem coleta. Esses resultados, todos de investigações realizadas em áreas peri- urbanas brasileiras, vêm reforçar as conclusões alcançadas nesta pesquisa.

Analisando cada vila separadamente, pode-se observar que, em geral, os valores de $O R$ não foram significativos, provavelmente em função do tamanho da amostra. Não é possível afirmar que há presença de risco na relação estudada.

O risco atribuível populacional, como mencionado anteriormente, foi determinado para os casos representados pelas diarréias, parasitoses e doenças dermatológicas e para os controles selecionados. Apesar dos valores relativamente baixos, os resultados da determinação do número de casos evitáveis nas regiões estudadas e da extrapolação dos valores obtidos para Belo Horizonte como um todo se mostraram bastante expressivos - 512 casos evitáveis nas sete vilas e favelas, considerando o quadro existente em 1995, e 2316 em Belo Horizonte, no ano de 1994 (tabela 7). Tais dados indicam a importância de se estender o atendimento por coleta de resíduos sólidos domiciliares a toda a população.

Neste contexto, deve-se ter uma certa cautela ao analisar os resultados, pois o estudo abrangeu apenas crianças moradoras de vilas e favelas. Ou seja, os resultados representam o número de casos que poderiam ser evitados em crianças com características semelhantes às das vilas e favelas, sendo os valores encontrados para o total da população infantil do município meramente ilustrativos. Obvia-

TABELA 7. Número de casos de diarréia, parasitoses e doenças dermatológicas que poderiam ser evitados em Belo Horizonte e nas regiões estudadas, Brasil, 1995

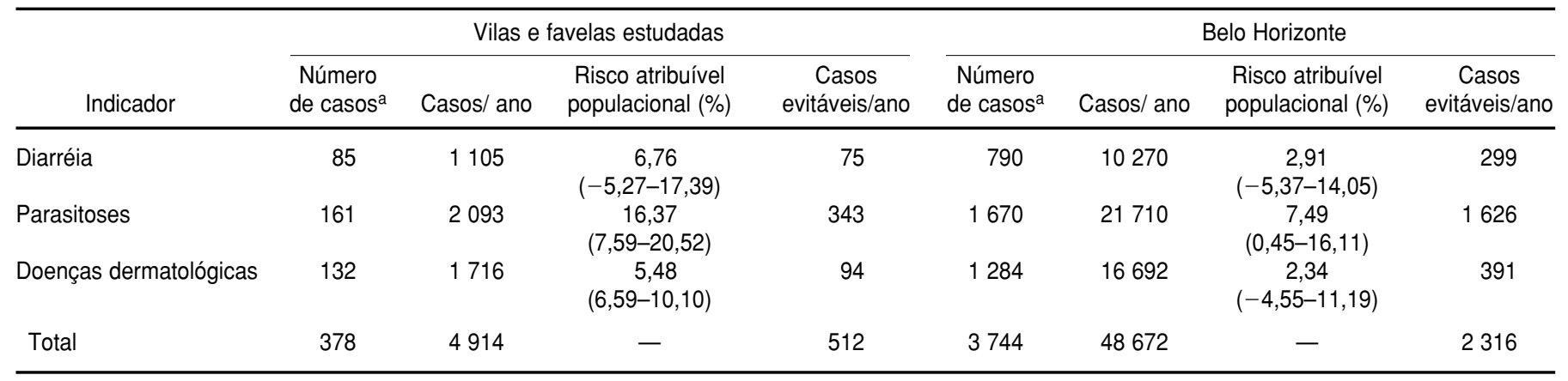

a Considerando o inquérito de 4 semanas realizado pela Secretaria Municipal de Saúde em 1994. 
mente, a interpretação do risco atribuível deve ser analisada de forma relativa, pois a mensuração de índices que indicam a quantidade de casos evitáveis através do aumento do atendimento por serviços de coleta simplifica os complexos determinantes do processo saúde-doença.

Quanto à verificação da influência do esgoto sanitário como variável de confusão, através da análise bivariada, deve-se reconhecer que a avaliação de somente um fator, deixando-se de conhecer a influência de outras possíveis variáveis de confusão, é limitada. Nessa análise, pode-se notar que, para o Aglomerado da Serra, ficou caracterizada a presença do fator de confusão em todas as hipóteses analisadas, enquanto que para o Aglomerado do Santa Lúcia os resultados indicaram confusão apenas quando as doenças dermatológicas foram incluídas como casos. No entanto, é provável que a presença do fator de confusão não tenha muito significado do ponto de vista da pesquisa realizada, uma vez que, para o resultado geral, em nenhum momento este efeito foi verificado. Neste contexto, os resultados encontrados para a amostra como um todo vêm reafirmar os resultados dos $O R$, indicando que o risco verificado é devido exclusivamente à medida de exposição analisada.

$\mathrm{Na}$ relação entre serviços de coleta de lixo e saúde, no entanto, diversos outros potenciais fatores de confusão podem ser cogitados, como escoamento de esgoto pelas ruas; presença de córregos nas vilas; forma de abastecimento, tipo de reservatório e qualidade da água servida à população; hábitos higiênicos pessoais e domésticos. Porém, a metodologia adotada dificulta a avaliação destes fatores, devido ao caráter retrospectivo do trabalho. Seria complexo coletar dados retrospectivos em relação a essas variáveis, pois seria pouco provável que se conseguisse reconstituir os cenários a elas referentes. Outra dificuldade reside na sistemática adotada - de coleta de dados secundários - a qual não permite a obtenção de informações rela- tivas a características individuais. Contudo, estudos epidemiológicos desenvolvidos na África e na Ásia sugerem que a consideração de variáveis de confusão em estudos sobre saneamento é, possivelmente, desnecessária, dado que estas variáveis alteram pouco os valores brutos das medidas de risco (18).

Como a seleção dos casos foi feita a partir do sistema de informações da Secretaria Municipal de Saúde e considerando-se que o processo de coleta de dados desse sistema é o mesmo para todas as regiões estudadas, assim como para todo o município de Belo Horizonte, aparentemente não se verificaram vícios ou diferenciações nessa seleção. Porém, podem ter ocorrido falhas de diagnóstico ou perdas na coleta de dados por parte da Secretaria. Seria impossível detectar estas falhas ou perdas, devido ao fato de ser este um estudo retrospectivo. Análise análoga pode ser desenvolvida para a caracterização das amostras de controles, uma vez que casos e controles originaram-se da mesma amostragem.

O fato da coleta de dados não ter sido rotineira, já que houve uma mobilização específica da rede municipal de saúde durante as 4 semanas de coleta de dados em 1994, tende a minimizar eventuais vícios. Além disso, considerando-se que, na sistemática de coleta de dados, os formulários cujas informações apresentaram dúvidas retornaram à fonte para reavaliação e posterior aproveitamento ou descarte, julgou-se que os dados são representativos e apresentam adequada qualidade, não devendo ter sido verificado viés nos resultados.

Outra avaliação necessária relaciona-se ao sistema de geoprocessamento, que apresenta deficiências quanto à localização dos endereços notificados. Tais deficiências devemse à desatualização da base contendo o município de Belo Horizonte, que se refere ao ano de 1989. Como pode ser observado na tabela 3, boa parte das perdas ocorreu em função deste problema no programa. Algumas vilas apresentaram perda mais significativa do que outras, refletindo dificuldades diferenciadas de localização das moradias entre as vilas. Em vista disso, há possibilidade deste fato ter provocado algum viés de seleção, não sendo possível, porém, verificar essa influência nos resultados. De qualquer modo, mesmo que o total de perdas $(23,14 \%)$ fosse relativo aos grupos de casos não-expostos e controles expostos, o valor de $O R$ ainda seria superior a 1,0 .

\section{CONCLUSÕES}

Embora pouco pesquisada, há uma indicação, no meio técnico, da existência de associação entre doenças infecciosas e parasitárias e o manejo ineficiente de resíduos sólidos. A revisão bibliográfica desta pesquisa constatou a exigüidade de dados quantitativos sobre a relação. O estudo epidemiológico desenvolvido revelou associação entre ausência de coleta de resíduos sólidos domiciliares e saúde pública, contribuindo para a sustentação científica desta relação.

Quantitativamente, o estudo indicou valores de $O R$ da ordem de 1,40, sugerindo que a população infantil exposta à ausência de serviços de coleta dos resíduos sólidos domiciliares possui $40 \%$ mais possibilidade de apresentar doenças diarréicas, parasitárias e dermatológicas do que a não exposta. Indicou ainda o estudo que a universalização deste importante serviço poderia evitar, considerando o quadro existente em 1995, 512 casos daquelas doenças nas crianças das vilas e favelas estudadas e, para a situação existente em 1994, de 2316 casos na população infantil de toda a cidade.

Em termos gerais, a metodologia se mostrou adequada. Contudo, algumas deficiências podem ter ocorrido, especialmente por se analisar um período retrospectivo e por se trabalhar com dados secundários. Para futuros estudos com o mesmo desenho, seria recomendável que a investigação se realizasse simultaneamente à coleta de dados; que pelo menos uma amostra de domicílios fosse visitada, com a 
aplicação de questionários; que os domicílios não localizados no sistema de geoprocessamento fossem identificados in loco; e que a coleta de dados não se restringisse a sistemas como os da Secretaria Municipal de Saúde de Belo Horizonte.
Agradecimentos. Os autores agradecem à Superintendência de Limpeza Urbana de Belo Horizonte por tornar disponíveis os dados relativos ao sistema de limpeza urbana pública e pelo financiamento de parte da pesquisa; à Secretaria Municipal de Saúde por

\section{REFERÊNCIAS}

1. Pereira Neto JT, Castilhos Júnior AB, Oliveira SML. Resíduos sólidos domiciliares: um paradoxo da sociedade moderna. Em: Associação Brasileira de Engenharia Sanitária e Ambiental (ABES). Anais do $17^{\circ}$ Congresso Brasileiro de Engenharia Sanitária e Ambiental. Natal: ABES; 1993:311-319.

2. Girout E, Brown JCA. Public health aspects of municipal solid waste management. Em: UNEP-International Environmental Technology Centre. International source book on environmentally sound technologies for municipal solid waste management. Osaka/Shiga: UNEP; 1996:395-406.

3. Risso WM. Aspectos relacionados à saúde ligados a resíduos sólidos. Em: Asociación Interamericana de Ingeniería Sanitaria y Ambiental (AIDIS). Anais do $1^{\circ}$ Simpósio Latino Americano de Resíduos Sólidos. São Paulo: AIDIS; 1993:16.

4. Fedorak PM, Rogers RE. Assessment of the potential health risks associated with the dissemination of microorganisms from a landfill site. Waste Manag Res 1991;9:537-563.

5. Najm ACM. Aspectos epidemiológicos. Em: Companhia de Tecnologia de Saneamento Ambiental. Gerenciamento de sistemas de resíduos sólidos. São Paulo: CETESB; sem data:1-25.

6. Lima LMQ. Tratamento de lixo. 2a ed. São Paulo: Helmus; 1991.
7. Pahren RH. Microorganisms in municipal solid waste and public health implications. CRC Crit Rev Environ Control 1987;3:187-228.

8. Cairncross S, Feachem RG. Environmental health engineering in the tropics: an introductory text. 2a ed. Chichester: John Wiley \& Sons; 1993.

9. Rajagopalan S. Guide to simple sanitary measures for the control of enteric diseases. Geneva: WHO; 1974, citado em: Feachem RG, McGarry M, Mara DD. Water, wastes and health in hot climates. 4a ed. Chichester: John Wiley \& Sons; 1986.

10. Mara DD, Alabaster GP. An environmental classification of housing-related diseases in developing countries. J Trop Med Hyg 1995; 98(1):41-51.

11. Briscoe J, Feachem RG, Rahaman MM. Evaluating health impact: water supply, sanitation, and hygiene education. Ottawa: International Development Research Centre; 1986.

12. Almeida Filho N, Rouquayrol MZ. Introdução à epidemiologia moderna. 2a ed. Belo Horizonte: COOPEMED; 1992.

13. Schlesselman JJ. Case control studies: design, conduct, analysis. New York: Oxford University Press; 1982.

14. Mantel N, Haenszel W. Statistical aspects of the analysis of data from retrospective studies of disease. J Natl Cancer Inst 1959;22: 719-748. tornar disponível a base cartográfica e pelo fornecimento de dados; e à Fundação de Capacitação de Pessoal Docente para o Ensino Superior (CAPES) pela concessão de bolsa de mestrado (CAAC).
15. Heller L. Associação entre cenários de saneamento e diarréia em Betim-MG : o emprego do delineamento epidemiológico caso controle na definição de prioridades de intervenção [tese de doutorado]. Belo Horizonte: Escola de Veterinária da Universidade Federal de Minas Gerais; 1995.

16. Rêgo, RCF. Destino dos dejetos, lixo e diarréia infantil em uma comunidade periurbana de Salvador, Bahia [dissertação de mestrado]. Salvador: Instituto de Saúde Coletiva da Universidade Federal da Bahia; 1996.

17. Moraes, LRS. Aspectos epidemiológicos relacionados aos resíduos sólidos domiciliares urbanos: um estudo de caso [CD-ROM]. Em: Associação Brasileira de Engenharia Sanitária e Ambiental (ABES). Anais do $19^{\circ}$ Congresso Brasileiro de Engenharia Sanitária e Ambiental. Foz do Iguaçu: ABES; 1997.

18. Briscoe J, Baltazar J, Young B. Case-control studies of the effect of environmental sanitation on diarrhoea morbidity: methodological implications of field studies in Africa and Asia. Int J Epidemiol 1988;17(2):441-447.

Manuscrito recebido em 7 de outubro de 1997. Aceito em versão revisada em 16 de novembro de 1998
ABSTRACT Association between
household solid waste
collection and health
There are few published studies about the effects of inadequate solid waste collection on the health of the population exposed to this situation. The objective of the present work was to describe this association in a sample of children under 5 years of age living in seven low-income neighborhoods and favelas in the city of Belo Horizonte, state of Minas Gerais, Brazil. We defined as "exposed" those children whose families were not served by waste collection; "not-exposed" were children who lived in areas with waste collection. The study employed data collected in 1994 and organized as a database by the municipal department of health. We employed a cross-sectional design, in which a "case" was defined as a child whose outpatient clinic record indicated a diagnosis of diarrheal, parasitic, or dermatological disease. Other diagnoses for the same age group composed the control group. Our epidemiological study revealed an association between the absence of domestic solid waste collection and public health. Our results suggest that the children exposed to the absence of solid waste collection have a $40 \%$ higher odds $(O R=1.40)$ of presenting diarrheal, parasitic, and dermatological diseases than not-exposed children. In addition, the calculation of attributable risk revealed that the presence of waste collection could prevent (based on the 1995 situation) 512 cases in the neighborhoods studied and (based on the 1994 conditions) 2316 cases among children in the entire city of Belo Horizonte. 